UDC 69.001.5

Author: KUDRYAVTSEV Pavel Gennadievich, Professor, D.Sc., Leading Researcher Polymate Israel Research Center (Israel), Academician of International Academy of Sciences for Ecology and Human Safety and Russian Academy of Natural Sciences, Author of 207 scientific works, including 4 monographs, has 34 inventions. He was awarded the M.V. Lomonosov medal, Commander of the Order «The Star of the Scientist» and was awarded the honorary title «Honored Worker of Science», awarded the medal of P.A.Stolypin, and honorable diamond sign of the Chamber of Commerce and Industry of the Russian Federation. Polymate Ltd. - Israel Research Center, POBox 73, Migdal HaEmek 10550, Israel, e-mail: pgkudr89@gmail.com;

Author: FIGOVSKY Oleg Lvovich, Professor, Full Member of European Academy of Sciences, Foreign Member of REA and RAASN, Editor-in-Chief of Journals SITA (Israel), OCJ and ICMS (USA), Director for Research and Development Polymate - Israel Research Center (Israel) and Nanotech Industries Inc., (USA); Chairman of the UNESCO chair «Green Chemistry»; President of Israel Association of Inventors; Laureate of the Golden Angel Prize, Commander of the Order «Engineering glory» (Russia) and «Leading Intellectuals of the World» (USA). Polymate Ltd. - Israel Research Center, POBox 73, Migdal HaEmek 10550, e-mail: figovsky@gmail.com

\title{
INVESTIGATION OF SOL-GEL TRANSITION BY RHEOLOGICAL METHODS. PART I. EXPERIMENTAL METHODS
}

\section{ExTENDEd Abstract:}

In this work rheological studies of the gelling process were carried out. We have developed a measuring system for studying the rheology of the gelation process. It consisted of several measuring cells of the Weiler-Rebinder type, system for automatic regulation of the composition of the medium and thermostabilization system. This complex is designed to measure the dependence of the value of the ultimate shear stress as a function of time, from the start of the sol-gel transition to the complete conversion of the sol to the gel. The developed device has a wide range of measured values of critical shear stresses $\tau_{0}=(0,05 \div 50000) \mathrm{Dyne} / \mathrm{cm}^{2}$. Using the developed instrument, it was possible to establish the shape of the initial section of the curve $\tau_{0}=f(t)$ and develop a methodology for more accurate determination of gelation time. The developed method proved that the classical method for determining the start time of the sol-gel transition using the point of intersection of the tangent to the linear part of the rheological curve $\tau_{0}=f(t)$, 
gives significantly distorted results. A new phenomenon has been discovered: the kinetic curves in the coordinates of the Avrami-Erofeev-Bogolyubov equation have an inflection point which separates the kinetic curve into two parts, the initial and the final. It was found that the constant $k$ in the Avrami-Erofeev-Bogolyubov equation does not depend on the temperature and is the same for both the initial and final parts of the kinetic curve. It depends only on the chemical nature of the reacting system. It was found that for the initial section of the kinetic curves, the value of the parameter $n$ in the Avrami-Erofeev-Bogolyubov equation was $n=23,4 \pm 2,8$ and, unlike the final section of the rheological curve, does not depend on temperature. A large value of this parameter can be interpreted as the average number of directions of growth of a fractal aggregate during its growth. The value of this parameter depends on the chemical nature of the sol particles participating in the gelling process. This behavior of the kinetic curves is due to a change in the mechanism of the process and a decrease in the number of possible directions for the growth of fractal aggregates. At the same time the process of aggregate growth gradually shifts from three-dimensional growth to twodimensional surface geometry, and even to a one-dimensional linear geometry. This phenomenon is due to steric hindrance arising from the aggregation of the remaining particles of sol in the formed gel. Based on rheological measurements, a sol-gel transition can be classified as a Second-order phase transition.

Key words: Sol-gel process, Rheology, Devices of the Weiler-Rebinder type, Kinetics of sol-gel transition, The Avrami-Erofeev-Bogolyubov equation, Secondorder phase transition.

MACHINE-READABLE INFORMATION ON CC-LICENSES (HTML-CODE) IN METADATA OF THE PAPER

$<$ a rel="license" href="http://creativecommons.org/licenses/by/4.0/" $><$ img alt="Creative Commons License" style="borderwidth:0" src="https://i.creativecommons.org/l/by/4.0/88x31.png" $/></ \mathrm{a}><$ br $/><$ span xmlns:dct="http://purl.org/ $\mathrm{dc} /$ terms/" href="http://purl.org/dc/dcmitype/Text" property="dct:title" rel="dct:type" $>$ Investigation of sol-gel transition by rheological methods. Part I. Experimental methods. $</$ span $>$ by $<$ a xmlns:cc $=$ "http://creativecommons.org/ ns\#" href="Nanotehnologii v stroitel'stve = Nanotechnologies in Construction. 2017, Vol. 9, no. 4, pp. 74-92. DOI: dx.doi. org/10.15828/2075-8545-2017-9-4-74-92. (In Russian)." property="cc:attributionName" rel="cc:attributionURL">K udryavtsev P.G., Figovsky O.L. $</ \mathrm{a}>$ is licensed under a $<$ a rel="license" href="http://creativecommons.org/licenses/ by $/ 4.0 / ">$ Creative Commons Attribution 4.0 International License $</ \mathrm{a}>$. $<$ br $/>$ Based on a work at $<$ a xmlns: dct="http:// purl.org/dc/terms/" href=" http://nanobuild.ru/en_EN/nanobuild-4-2017/" rel="dct:source" > http://nanobuild.ru/ en_EN/nanobuild-4-2017/</a $>$. $<$ br $/>$ Permissions beyond the scope of this license may be available at $<$ a xmlns:cc="http: $/ /$ creativecommons.org/ns\#" href="figovsky@gmail.com" rel="cc:morePermissions">figovsky@gmail.com</a> 
The part 2 of the paper by Kudryavtsev P.G., Figovsky O.L. «Investigation of solgel transition by rheological methods» will be published in the issue $5 / 2017$ in the Internet-Journal «Nanotechnologies in Construction».

The references for the part 1 and part 2 of the paper by Kudryavtsev P.G., Figovsky O.L. "Investigation of sol-gel transition by rheological methods» will be also published in the issue 5/2017 in the Internet-Journal «Nanotechnologies in Construction".

\section{DEAR COLLEAGUes!}

THE REFERENCE TO THIS PAPER HAS THE FOLLOWING CITATION FORMAT:

Kudryavtsev P.G., Figovsky O.L. Investigation of sol-gel transition by rheological methods. Part I. Experimental methods. Nanotehnologii v stroitel'stve = Nanotechnologies in Construction. 2017, Vol. 9, no. 4, pp. 74-92. DOI: dx.doi. org/10.15828/2075-8545-2017-9-4-74-92. (In Russian).

Contact information
Figovsky Oleg Lvovich

figovsky@gmail.com 
уДК 69.001.5

Автор: КУДРЯВЦЕВ Павел Геннадьевич, проф., D.Sc., ведущий научный сотрудник Polymate Israel Research Center (Израиль), действительный член (академик) МАНЭБ и РАЕН, автор 207 научных работ, в том числе 4 монографий, имеет 34 изобретения, награжден медалью им. М.В. Ломоносова, кавалер ордена «Звезда Ученого», удостоен почетного звания «Заслуженный деятель науки», награжден медалью П.А. Столыпина и почетным бриллиантовым знаком Торговопромышленной палаты РФ; POBox 73, Migdal HaEmek, Polymate Ltd. - Israel Research Center, 10550, Израиль, e-mail: pgkudr89@gmail.com;

Автор: ФИГОВСКИЙ Олег Львович, проф., действительный член Европейской академии наук, иностранный член РИА и РAACH, главный редактор журналов SITA, OCJ и RPCS, директор по науке и развитию Polymate - Israel Research Center (Израиль) и Nanotech Industries Inc. (USA), зав. каф. ЮНЕСКО «Зелёная химия», президент Израильской ассоциации изобретателей, лауреат Golden Angel Prize, кавалер орденов «Инженерная слава» (Россия) и «Leading Intellectuals of the World» (USA); POBox 73, Migdal HaEmek, Polymate Ltd. - Israel Research Center, 10550, Израиль, e-mail: figovsky@gmail.com

\section{ИССЛЕДОВАНИЕ ЗОЛЬ-ГЕЛЬ ПЕРЕХОДА РЕОЛОГИЧЕСКИМИ МЕТОДАМИ. ЧАСТЬ І. МЕТОДЫ ПРОВЕДЕНИЯ ЭКСПЕРИМЕНТОВ}

АННОТАЦИЯ К СТАТЬЕ (АВТОРСКОЕ РЕЗЮМЕ, РЕФЕРАТ):

В настоящей работе были проведены реологические исследования процесса гелеобразования. Для исследования реологии гелеобразования нами был разработан измерительный комплекс, состоящий из нескольких измерительных ячеек типа Вейлера-Ребиндера, системы автоматического регулирования состава среды и системы термостабилизации. Данный комплекс предназначен для измерения зависимости величины предельного напряжения сдвига от времени, с момента начала золь-гель перехода до полного превращения золя в гель. Разработанная установка имеет широкий диапазон измеряемых величин предельных напряжений сдвига $\tau_{0}=(0,05 \div 50000) \partial u н / c \mu^{2}$. Использование разработанного прибора позволило установить вид начального участка кривой $\tau_{0}=f(t)$ и разработать методику более точного определения времени гелеобразования. С использованием разработанного метода было установлено, что классический метод определения времени начала золь-гель перехода по точке пересечения касательной к линейной части кривой $\tau_{0}=f(t)$ 
дает значительно искаженные результаты. Обнаружено новое явление, которое заключается в том, что кинетические кривые в координатах уравнения Аврами-Ерофеева-Боголюбова имеют точку перегиба, которая разделяет кинетическую кривую на две части, начальную и конечную. Было обнаружено, что константа $\mathrm{k}$ в уравнении Аврами-Ерофеева-Боголюбова не зависит от температуры и одинакова, как для начальной, так и конечной части кинетической кривой. Она зависит только от химической природы реагирующей системы. Было обнаружено, что для начального участка кинетических кривых величина параметра $\mathbf{n}$ в уравнении Аврами-Ерофеева-Боголюбова составила $n=23,4 \pm 2,8$ и, в отличие от конечного участка реологической кривой, не зависит от температуры. Болышая величина этого параметра может быть интерпретирована как среднее число направлений роста фрактального агрегата в процессе его роста. Величина этого параметра зависит от химической природы частиц золя, участвующих в процессе гелеобразования. Обнаруженное поведение кинетических кривых обусловлено изменением механизма процесса и уменьшением числа возможных направлений роста фрактальных агрегатов. При этом процесс роста агрегатов постепенно переходит от трехмерного роста к двухмерной поверхностной геометрии и даже к одномерной линейной. Как было показано, это обусловлено стерическими затруднениями, возникающими при агрегации оставшихся частиц золя в сформированном геле. Опираясь на реологические измерения, золь-гель переход можно классифицировать как фазовый переход II рода.

Ключевые слова: золь-гель процесс; реология; приборы типа ВейлераРебиндера; кинетика золь-гель-перехода; уравнение Аврами-Ерофеева-Боголюбова; фазовый переход II рода.

DOI: dx.doi.org/10.15828/2075-8545-2017-9-4-74-92

\footnotetext{
МАШИНОЧИТАЕМАЯ ИНФОРМАЦИЯ О СС-ЛИЦЕНЗИИ В МЕТАДАННЫХ СТАТЬИ (HTML-КОД):

$<$ a rel="license" href="http://creativecommons.org/licenses/by/4.0/"><img alt="Лицензия Creative Commons" style="borderwidth:0" src="https://i.creativecommons.org/l/by/4.0/88x31.png" / $></ \mathrm{a}><$ br $/>$ Произведение "<span xmlns:dct="http:// purl.org/dc/terms/" href="http://purl.org/dc/dcmitype/Text" property="dct:title" rel="dct:type">Исследование золь-гель перехода реологическими методами. Часть I. Методы проведения экспериментов </span>» созданное автором по имени $<$ a xmlns:cc="http://creativecommons.org/ns\#" href="Нанотехнологии в строительстве. - 2017. - Том 9, № 4. - С. 74-92. - DOI: dx.doi.org/10.15828/2075-8545-2017-9-4-74-92." property="cc:attributionName" rel="cc:attributionURL"> Кудря вцев П.Г., Фиговский О.Л.</a>, публикуется на условиях <a rel="license" href="http://creativecommons.org/licenses/ by/4.0/">лицензии Creative Commons «Attribution» («Атрибуция») 4.0 Всемирная $</ \mathrm{a}>$. $<\mathrm{br} />$ Основано на произведении с <a xmlns:dct="http://purl.org/dc/terms/" href="http://nanobuild.ru/ru_RU/nanobuild-4-2017/" rel="dct:source">http:// nanobuild.ru/ru_RU/nanobuild-4-2017/</a >.<br />Разрешения, выходящие за рамки данной лицензии, могут быть доступны на странице <a xmlns:cc="http://creativecommons.org/ns\#" href="figovsky@gmail.com" rel="cc:morePermissions">figovs $\mathrm{ky} @$ gmail.com $</ \mathrm{a}>$.
} 


\section{Введение}

Одним из новых и перспективных путей создания современных наноструктурированных строительных материалов являются процессы, основанные на золь-гель технологии [1]. В основе золь-гель технологии лежит важнейшая особенность коллоидных растворов (золей) - способность к образованию геля. Гелеобразование обеспечивает возможность широкого применения коллоидных растворов в качестве связующих при получении композитов, носителей катализаторов, сорбентов и т.д.

Образованию гелей предшествует индукционный период, в течение которого не наблюдается существенного изменения свойств коллоидной системы. После завершения индукционного периода резко растет вязкость коллоидной системы, после чего наступает потеря текучести. Продолжительность индукционного периода также обычно называется временем гелеобразования и относится к кинетической характеристике этого процесса. Во время индукционного периода в коллоидных растворах в результате процесса агрегации формируются зародыши новой фазы, происходит их рост и коагуляция. Эти процессы предшествуют образованию «бесконечного» агрегата - геля.

Переход золя в гель связан с образованием пространственных цепочек, которые пронизывают весь объем золя. Возникновение пространственной сетки сопровождается значительным возрастанием вязкости системы. У нее появляется ряд новых физических свойств: упругость, предельные напряжения сдвига, способность к сохранению формы. Коллоидная система утрачивает текучесть под воздействием собственной массы и малых внешних напряжений.

Вязкость и предел текучести - это две макроскопические интегральные характеристики ньютоновской (золь) и бингамовской (гель) сред, входящие в реологические уравнения. Существует много различных методов измерения вязкости, которые могут быть объединены в следующие группы: а) методы капиллярной вискозиметрии; б) методы ро- 
тационной вискозиметрии; в) методы вибрационной вискозиметрии; г) метод падающего шарика; д) методы пластометрии. Достаточно полное описание методов измерения вязкости и приборов, используемых для этих целей, содержится в работе [2].

Известны также различные способы определения времени гелеобразования. Например, методы «падающей палочки», падающего шарика, смещения мениска, которые состоят в визуальном наблюдении потери текучести золя и не могут дать удовлетворительной точности и достоверности измерений времени гелеобразования. Определение времени гелеобразования обычно осуществляется путем измерения предельных напряжений сдвига. Для этих целей обычно применялся метод тангенциального сдвига пластинки [4], реализуемый при помощи приборов типа Вейлера-Ребиндера [3], однако существует и ряд других методов.

Одним из таких примеров является метод погружения капилляра [5], который представлен ниже на рис. 1.

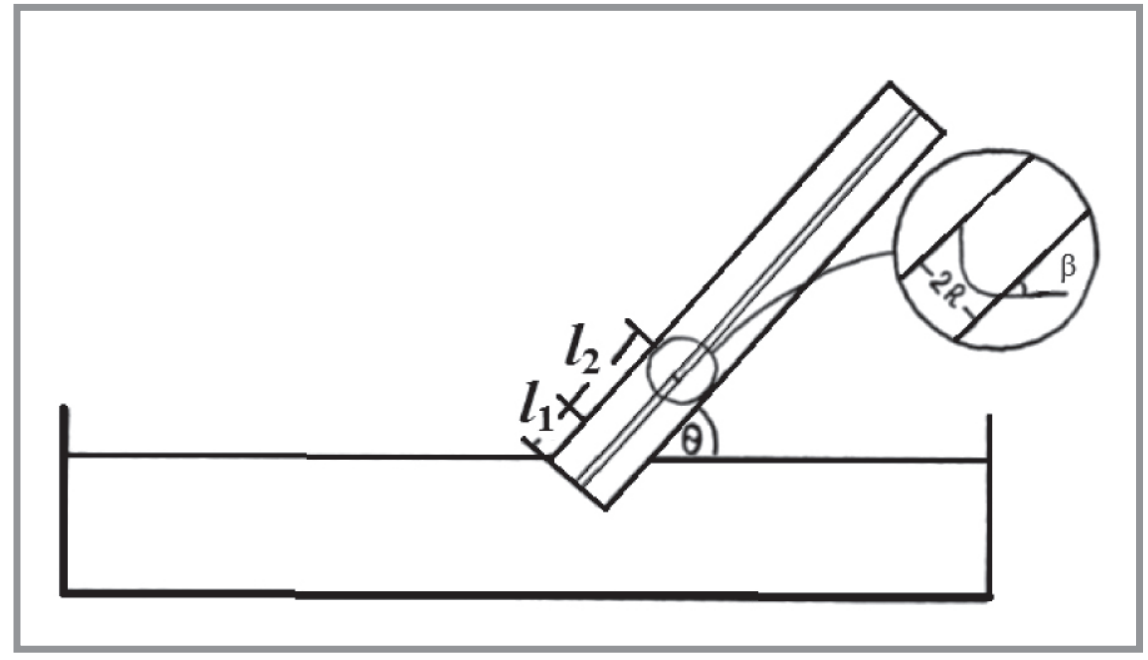

Рис. 1. Измерение предельных напряжений сдвига методом погружения капилляра: $l_{2} \sin \theta$ - высота подъема золя по капилляру; $l_{1}$ - длина капилляра ниже поверхности золь-воздух

На этом примере рассмотрим поведение ньютоновских и неньютоновских жидкостей в капиллярном вискозиметре. Равновесное состояние ньютоновской жидкости, заполняющей капилляр, характеризуется балансом сил поверхностного натяжения и гравитации. Для конфигурации, изображенной на рис. 1 , баланс сил записывается в виде: 
$2 \sigma \pi R \cos \beta=g \pi R^{2} l_{2} \sin \theta$,

где $R$ - радиус капилляра; $\sigma$ - коэффициент поверхностного натяжения; $\beta$ - угол смачивания; $\rho$ - плотность жидкости; $g$ - ускорение свободного падения.

Если среда обладает предельным напряжением сдвига, то в уравнении баланса необходимо учесть дополнительную силу, препятствующую заполнению капилляра. Для случая слабо структурированной бингамовской среды уравнение (1) примет следующий вид:

$2 \sigma \pi R \cos \beta=g \pi R l_{2} \sin \theta+2 \tau_{0} \pi R\left(l_{1}+l_{2}\right)$,

где $\tau_{0}-$ предельное напряжение сдвига.

Следовательно, величина предельного напряжения сдвига может быть найдена из выражения:

$$
\tau_{0}=\frac{2 \sigma \cos \beta-\rho g R l_{2} \sin \theta}{2\left(l_{1}+l_{2}\right)} .
$$

Данным методом могут быть измерены сравнительно малые величины предельных напряжений сдвига. Недостатком метода можно считать необходимость тщательного обезжиривания внутренней поверхности капилляра. Эволюцию предельных напряжений сдвига с течением времени можно определить при помощи ротационного вискозиметра, но вследствие малой чувствительности прибора начальная часть реологической кривой не может быть определена. Метод тангенциального сдвига рифленой пластинки не включает в себя операций тщательного обезжиривания, а его чувствительность определяется чувствительностью используемых весов и площадью рифленой пластинки.

\section{Экспериментальная часть}

\section{1. Методы исследования}

Для реологических исследований процесса гелеобразования нами был разработан измерительный комплекс, состоящий из следующих блоков:

а) трех измерительных ячеек типа Вейлера-Ребиндера; 
б) блока автоматического изменения состава среды;

в) блока термостабилизации.

Данный комплекс предназначен для измерения зависимости величины предельного напряжения сдвига от времени, с момента начала золь-гель перехода до полного превращения золя в гель. Измерительная ячейка состоит из термостатирующей рубашки, индикатора и динамометра, в качестве которого используются торсионные весы. Блок автоматического изменения состава среды включает в себя иономер с электродами, блок сопряжения и автоматическую бюретку. Индентор выполнен в виде алюминиевой прямоугольной пластины, толщиной 0,1 мм и прикрепленной к тяге, которая изготовлена из волокна на основе из нитрида бора.

Схема разработанного прибора приведена на рис. 2. Блок 1 включает в себя приборы a, b, c, d: a - иономер, b - блок сопряжения, c - aвтоматическая бюретка, $\mathrm{d}$ - термостатируемая кювета, в которой автоматически, до заданной величины, изменяется $\mathrm{pH}$ золя. Блок 2 состоит из термостата (t), соединительных патрубков и рубашек для термостатирования трех кювет $(\mathrm{k}, \mathrm{l}, \mathrm{m})$. Измерительный блок 3 состоит из трех приборов типа Вейлера-Ребиндера разной чувствительности (f, g, h);

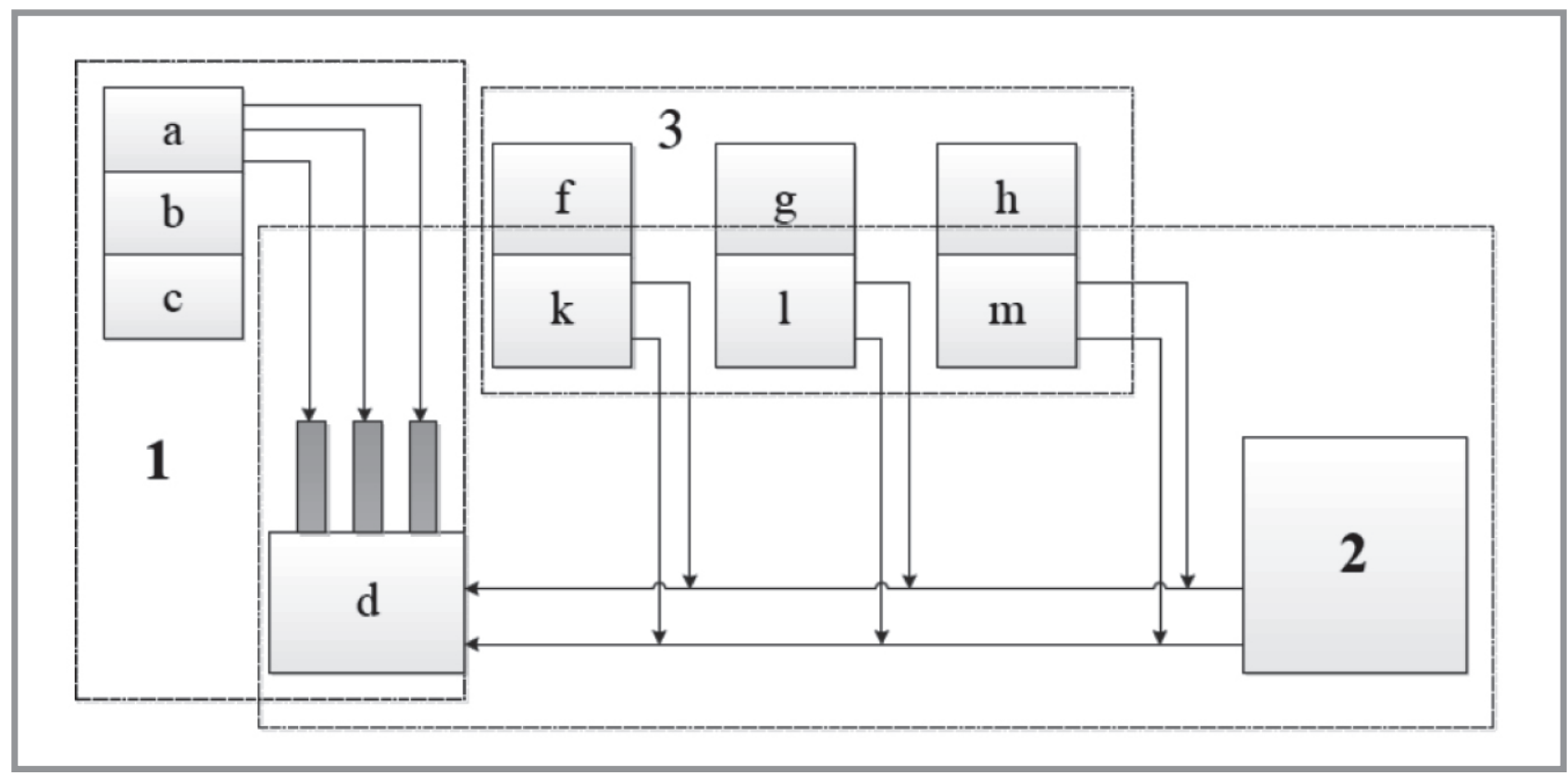

Рис. 2. Схема прибора для измерения предельных напряжений сдвига. (Описание в тексте) 
трех модернизированных торсионных весов и трех термостатируемых кювет. Наличие трех измерительных ячеек необходимо для полноты охвата всего измерительного диапазона предельных напряжений сдвига. Первая ячейка (f) работает в диапазоне $\tau_{0}=(0,05 \div 10,0)$ дин $/ \mathrm{cm}^{2}$, вторая $(\mathrm{g})-\tau_{0}=(5,0 \div 1000)$ дин $/ \mathrm{cm}^{2}$, а третья (h) $-\tau_{0}=(500 \div 50000)$ дин $/ \mathrm{cm}^{2}$.

Разработанный нами измерительный комплекс позволяет вести определения предельных напряжений сдвига в диапазоне значений $\tau_{0}=$ $(0,05 \div 50000)$ дин $/ \mathrm{cm}^{2}[6]$.

\section{2. Реагенты}

Для испытания измерительного комплекса для исследования процесса гелеобразования реологическими методами в качестве модельного объекта нами был использован кремнезоль с концентрацией 2\% $\mathrm{SiO}_{2}$, полученный ионообменным способом, описанным в работе [11]. Для стабилизации используемого кремнезоля, в его состав добавлялась концентрированная соляная кислота, до рН 1,8. Неизменность свойств золя контролировалась в течение всего эксперимента. Для инициирования процесса гелирования порция золя объемом 100 мл дестабилизировалась путем добавления $1,0 \mathrm{~N}$ раствора $\mathrm{NaOH}$, до $\mathrm{pH} 6,0$. Процесс проводился при различных температурных значениях реакционной среды. При проведении экспериментов мы поддерживали постоянными следующие параметры: $\mathrm{pH}$, концентрация и форма функции распределения частиц золя по размеру .

\section{3. Методика измерения}

При вертикальном смещении тонкой рифленой пластины, площадь поверхности которой равна $S$, под действием силы $F$ величина напряжений сдвига при тангенциальном сдвиге вязкой среды определяется выражением:

$$
\tau=\frac{F}{S} .
$$

Если при вытягивании пластинки индентора из геля мы будем плавно увеличивать величину приложенной силы, начиная от нулевого зна- 
чения, то сдвиг произойдет при некотором критическом значении этой силы $F^{*}$. В данном случае полученная величина напряжения сдвига $\tau_{0}$ имеет смысл предельного напряжения сдвига:

$$
\tau_{0}=\frac{F^{*}}{S},
$$

где $F^{*}$ - минимальная величина приложенной силы, при достижении которой наблюдается разрушение контакта пластина-гель, $S$ - площадь пластины.

В случае идеального тела Бингама достаточно произвести сдвиг пластины на бесконечно малое расстояние, после чего происходит разрушение контакта пластина-гель. В реальном эксперименте невозможно достичь таких условий. Кроме того, ситуация осложняется тем, что гель обладает определенной эластичностью, и разрушение контакта происходит при перемещении пластины на некоторое конечное расстояние, которое зависит от множества факторов. Такими влияющими факторами являются: стадии зрелости золя, концентрации золя и электролитного фона, температура, $\mathrm{pH}$ среды и т.д. Для того, чтобы преодолеть эти сложности, была исследована зависимость величины напряжений сдвига от длины перемещения пластинки ( $r$ ) (рис. 3). Как показал экс-

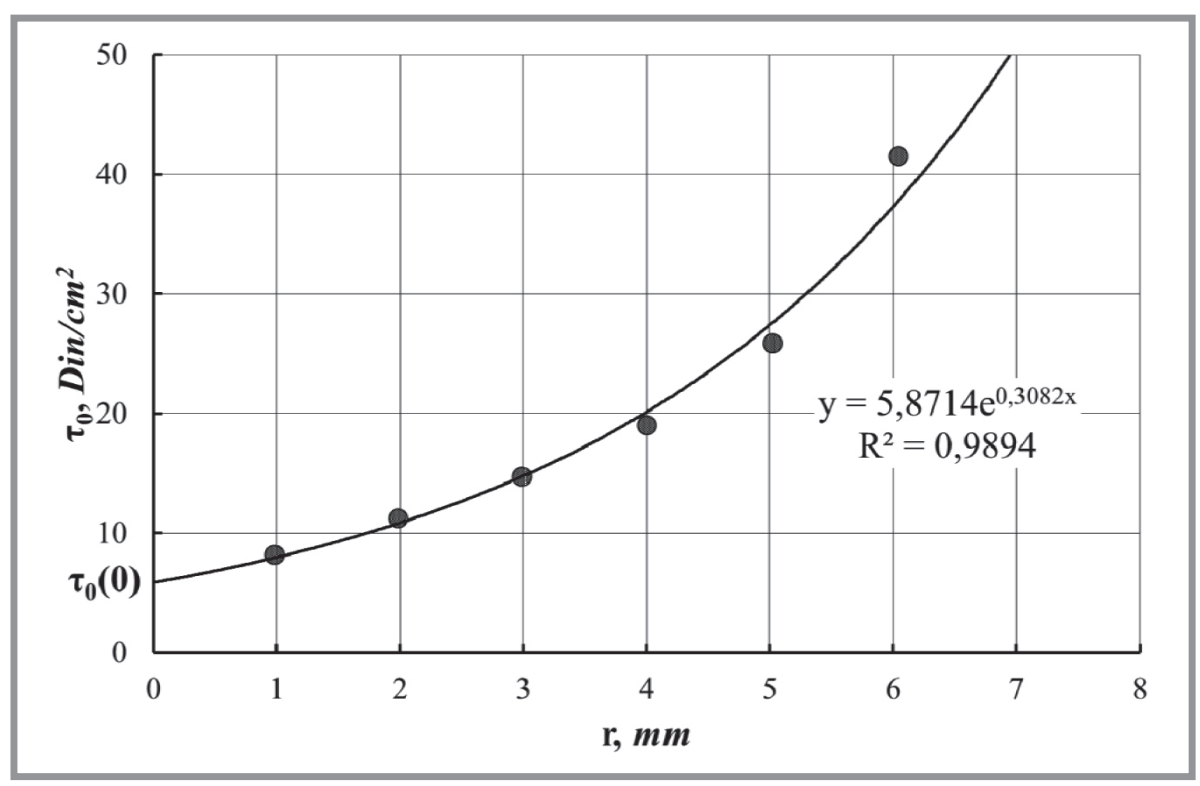

Рuc. 3. Зависимость величины напряжений сдвига от длины перемещения пластинки 
перимент, независимо от площади поверхности пластинки кривая $\tau_{0}(r)$ может быть описана уравнением (6):

$$
\tau_{0}=A \exp (B \cdot r)
$$

Определив в этом уравнении коэффициенты А и В, можно рассчитать величину $\tau_{0}$ при нулевом перемещении индентора $(r=0)$. Полученная величина имеет смысл предельных напряжений сдвига или предела текучести данной среды.

В работе [1] для определения времени гелеобразования использовался прибор типа Вейлера-Ребиндера с чувствительностью $\Delta=10$ дин $/ \mathrm{cm}^{2}$. Для того, чтобы приборная погрешность при такой чувствительности не превышала $5 \%$, необходимо измерять предельные напряжения сдвига, начиная с 200 дин $/ \mathrm{cm}^{2}$ (см. рис. 4.) Однако при величине предельных напряжений сдвига, равных $5 \div 10$ дин $/ \mathrm{cm}^{2}$, потерю текучести золя можно уже определить визуально, например, методом смещения мениска. Таким образом, для детального исследования реологической кривой необходимо было иметь прибор, имеющий нижний предел измерения предельных напряжений сдвига около $\tau_{\min }=0,05$ дин $/ \mathrm{cm}^{2}$. При этом порог чувствительности прибора должен быть не хуже, чем $\Delta \leq 0,005$ дин $/ \mathrm{cm}^{2}$.

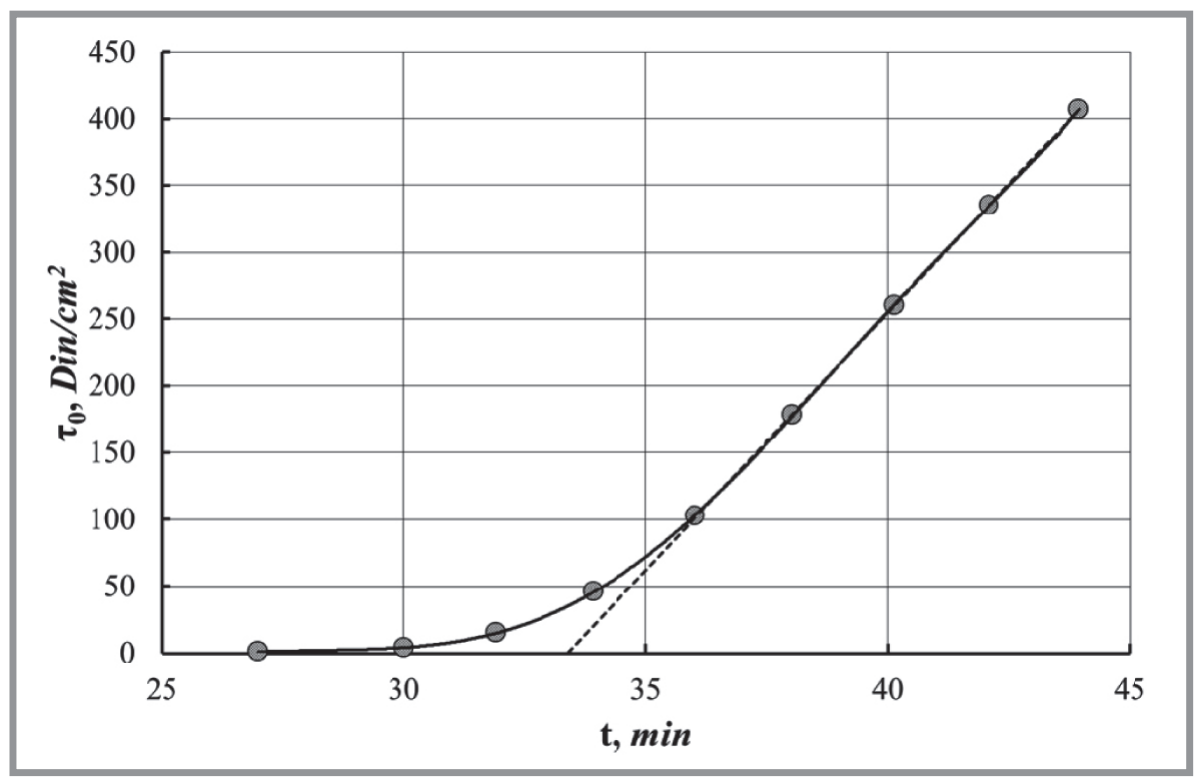

Puc. 4. Вид начальной части реологической кривой $\tau_{0}(t)$ при гелировании разбавленных золей 
Порог чувствительности прибора определяется нижним пределом измерения силы $\left(P_{\min }=0,25\right.$ дин). Он характеризуется типом используемых торсионных весов и площадью поверхности индентора $\left(S=50 \mathrm{~cm}^{2}\right)$. Такие параметры были достигнуты на разработанном нами приборе. Отсюда стабильные измерения предельных напряжений сдвига достигаются в соответствии с формулой (4), когда порог чувствительности составляет величину меньше, чем относительная погрешность измерений $\delta<10 \%$.

Важным аспектом таких реологических экспериментов является методика определения времени начала гелеобразования. Так, в работе [4] время гелеобразования определялось по точке пересечения касательной к линейной части кривой $\tau_{0}(t)$ с осью абсцисс.

Как показали эксперименты, проведенные на разработанном приборе, начальный участок кривой $\tau_{0}(t)$ имеет вид, представленный на рис. 4 (сплошная линия).

Начальный участок экспериментальной зависимости $\tau_{0}(t)$ хорошо описывается уравнением:

$$
\tau_{0}=B \exp (C \cdot t)+D .
$$

Параметры B, C, D в уравнении (7) могут быть определены численно методом наименьших квадратов по экспериментальным значениям $\tau_{0}=f(t)$. Время гелеобразования $t^{*}$ определяется из выражения (7) при условии $\tau_{0}=0$ :

$$
t^{*}=\frac{1}{C} \ln \left(-\frac{D}{B}\right) .
$$

Описанный выше способ определения времени гелеобразования представляется более корректным, чем описанный в работе [4]. Использование разработанного нами прибора позволило установить более детальный вид начального участка кривой $\tau_{0}=f(t)$ и разработать методику более точного определения времени гелеобразования, что имеет большое значение при получении неорганических композитов по зольгель технологии. 


\section{4. Метод определения времени гелеобразования с использованием реологических методов}

Настоящий раздел посвящен определению времени гелеобразования с использованием реологических методов. Экспериментально была исследована зависимость предельных напряжений сдвига и предела текучести от времени при гелировании кремнезоля. Для того, чтобы дать определение времени гелеобразования, предварительно необходимо обсудить ряд условий, при соблюдении которых это определение имело бы наиболее простой вид и могло бы быть использовано экспериментатором.

При изучении зависимости времени гелеобразования от какого-либо параметра или для определения погрешности измерения времени гелеобразования, необходимо проводить ряд изменений в течение какогото времени $t^{\prime}$. Очевидно, что в течение этого времени свойства исходного золя должны быть постоянны. Для этого золи стабилизируют различными способами, изменяя $\mathrm{pH}$, температуру, вводя химические добавки. Чтобы характеризовать постоянство или изменение свойств золя, необходимо измерять временную эволюцию какого-либо его интегрального макропараметра, например, эффективной вязкости [7].

При неизменности свойств золя должно выполняться следующее условие:

$$
K=\frac{\eta\left(t^{\prime}\right)-\eta(0)}{\eta(0)} \ll 1,
$$

где $\eta(0)$ - эффективная вязкость исходного золя; $\eta\left(t^{\prime}\right)$ - эффективная вязкость золя, хранившегося время $t^{\prime}$.

Для дестабилизации определенного количества исходного золя требуется некоторое время $t^{\prime \prime}$. Продолжительность этой стадии $t^{\prime \prime}$ зависит от объема дестабилизируемого золя, его $\mathrm{pH}$, скорости титрования корректирующим раствором и конечного $\mathrm{pH}$ титруемого раствора золя. Важным является вопрос начала отсчета при измерении времени протекания процесса гелеобразования. Использовать в качестве начала отсчета точку конца титрования не совсем корректно. Это связано с тем, что изменение $\mathrm{pH}$ до заданной величины, особенно в конце титрования, происходит медленно. Такой эффект создает неопределенность при фиксации точки начала отсчета. Начало титрования является строго 
определенным моментом времени, и использовать его в качестве начала отсчета значительно удобней. Некоторая погрешность, возникающая при этом, является постоянной величиной и зависит только от объема экспериментальной ячейки.

Временем гелеобразования обычно называют временной интервал, отсчитываемый от момента времени, соответствующего началу гелеобразования, до момента времени, когда гелирующий золь потеряет свою текучесть.

Момент потери текучести определяют различными способами. Например, методом отслеживания изменений формы поверхности мениска, методом падающей палочки, методами вискозиметрии при стремлении эффективной вязкости к бесконечности [7], методом тангенциального сдвига пластинки. В последнем определяется зависимость предельных напряжений сдвига от времени $\tau(t)$.

При различных временах дестабилизации определенного количества исходного золя $t^{\prime}$ и $t^{\prime \prime}$, кривая $\tau_{0}(t)$ может сместиться на некоторый временной интервал $\Delta t$. При определении времени гелеобразования этим временем $\Delta t$ можно пренебречь, если выполняется условие:

$$
\frac{\tau\left(t^{\prime}+\Delta t\right)-\tau_{0}(t \prime)}{\tau_{0}(t \prime)} \ll 1
$$

где $\tau_{0}\left(t^{\prime}\right)$ - предельные напряжения сдвига в момент времени, соответствующие началу линейного участка кривой $\tau_{0}\left(t^{\prime}\right)$;

$\tau\left(t^{\prime}+\Delta t\right)-$ предельные напряжения сдвига при смещении по времени на $\Delta t$.

Это условие выполняется не всегда, поэтому для того, чтобы эксперимент мог быть кем-либо воспроизведен, необходимо, чтобы кроме прочих условий, была указана зависимость объема оттитрованного раствора в зависимости от времени или зависимость скорости титрования от времени. Характерный вид этих зависимостей представлен на рис. 5. Такой вид кривых титрования реализуется только в случае режима автоматического титрования в соотношении (9) величина коэффициента $K \leq 0,04$. При этом для каждой порции золя режим дестабилизации раствора сохранялся неизменным. Для каждого значения времени измерения всех величин проводились минимум три раза. Время гелеобразования было определено из реологических кривых методом наименьших квадратов. 


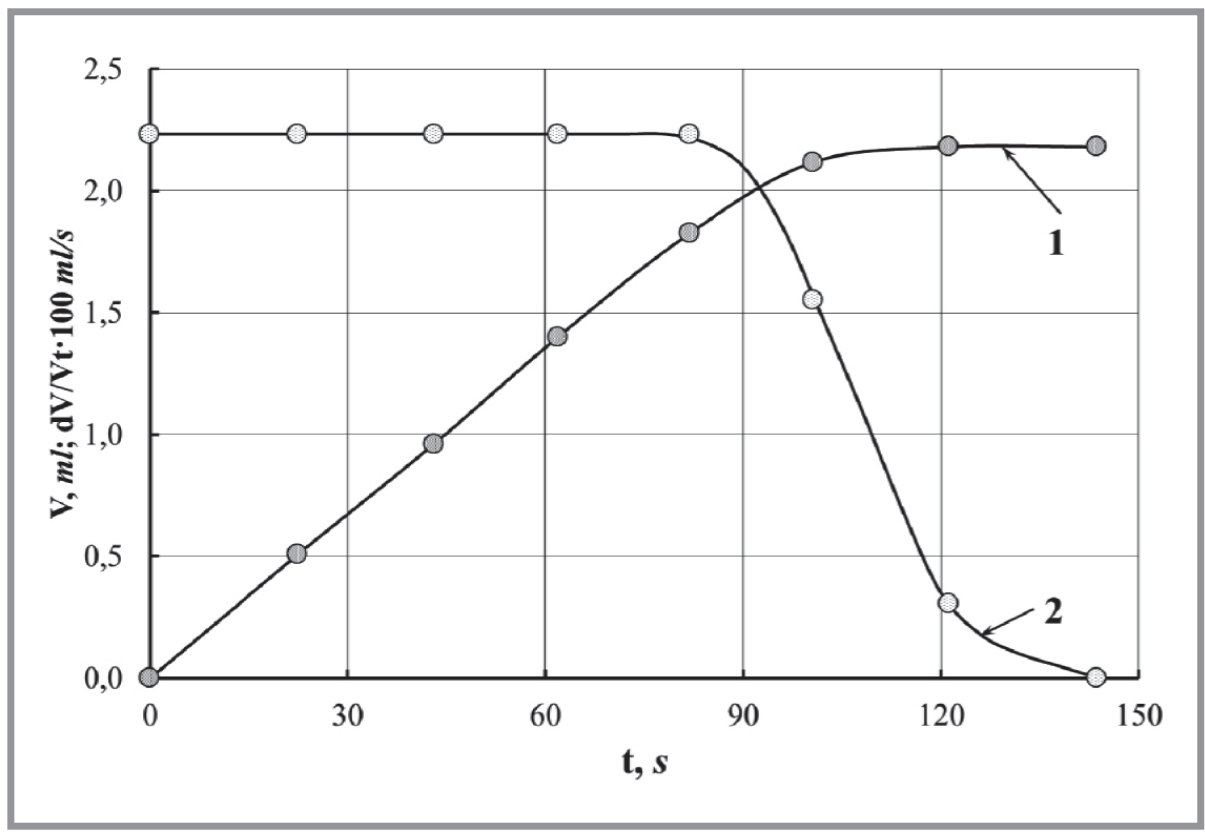

Рuc. 5. Зависимость объема (1) и скорости (2) дозировки раствора $\mathrm{NaOH}$ от времени

Проведенные на разработанном приборе эксперименты показали, что начальный участок кривой $\tau_{0}=f(t)$ имеет вид, представленный на рис. 6. Полученную экспериментальную зависимость величины предельных напряжений сдвига от времени можно условно разделить на три участка - нулевой, начальный и конечный. Нулевой участок приходится на интервал времени от инициирования процесса гелеобразования до момента появления у среды отличных от ноля предельных напряжений сдвига, соответствующих времени начала гелеобразования $t_{1}{ }^{*}$. На этом участке гелирующий золь характеризуется как классическая ньютоновская жидкость. Начальный участок реологической кривой расположен в интервале времени от начала гелеобразования $t_{1}{ }^{*}$ до точки завершения процесса гелеобразования. Конечный участок реологической кривой расположен в интервале времени от точки завершения процесса гелеобразования до точки полного завершения процесса гелеобразования, сопровождающегося упрочнением геля и выходом реологической кривой на постоянные значения предельных напряжений сдвига. На начальном и конечном участках реологической кривой материал характеризуется уже как неньютоновская жидкость или тело Бингама. 


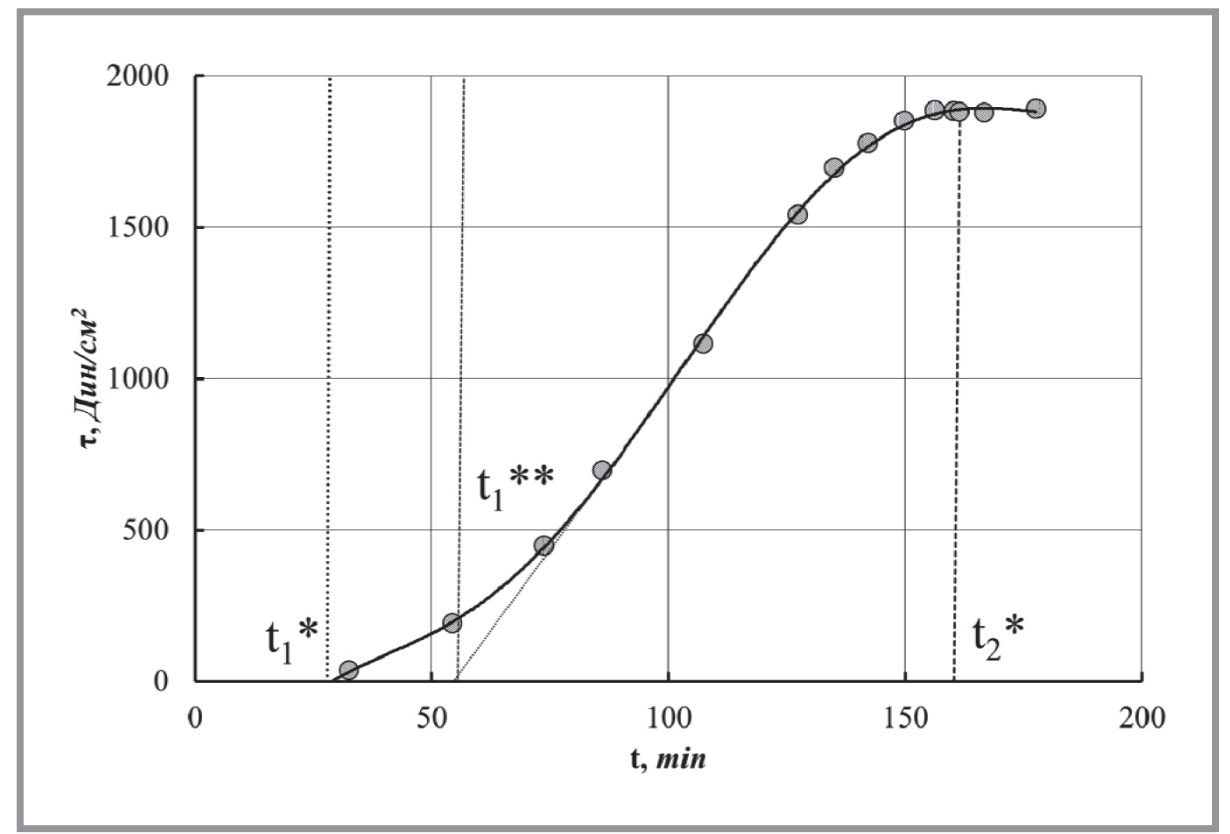

Рuc. 6. Вид полной кривой зависимости предельных напряжений сдвига от времени $\tau_{0}=f(t)$ при гелировании кремнезоля с концентрацией $3 \%$, при $\mathrm{pH}=6,0$ и $\mathrm{T}=20^{\circ} \mathrm{C}$

Экспериментальные данные для начального участка зависимости предельных напряжений сдвига от времени хорошо описываются следующим уравнением:

$$
\tau_{0}=A_{1} \exp \left(B_{1} t\right)+C_{1} .
$$

Эмпирические параметры $\mathrm{A}_{1}, \mathrm{~B}_{1}, \mathrm{C}_{1}$ в этом уравнении могут быть определены численно методом наименьших квадратов. Время начала гелеобразования $t_{1}{ }^{*}$ определяется из выражения (11) при $\tau_{0}=0$ :

$$
t_{1}^{*}=\frac{1}{B_{1}} \ln \left(-\frac{C_{1}}{A_{1}}\right) \text {. }
$$

Экспериментальные данные для конечного участка зависимости предельных напряжений сдвига от времени хорошо описываются следующим уравнением:

$$
\tau_{0}=A_{2} \ln \left(B_{2} t\right)+C_{2} \text {. }
$$


Коэффициенты $\mathrm{A}_{2}, \mathrm{~B}_{2}, \mathrm{C}_{2}$ могут быть определены аналогично, как для начального участка. Время полного превращения золя в гель равно $t_{2}{ }^{*}$, определяется из выражения (13) при $\tau_{0}=\tau_{\max }$ :

$$
t_{2}^{*}=\frac{1}{B_{2}} \exp \left\{\frac{\tau_{\max }-C_{2}}{A_{2}}\right\} .
$$

В момент времени $t_{1}$, , соответствующему началу гелеобразования, в гелирующем золе образуется кластер, края которого касаются стенок сосуда, т.е. среда начинает проявлять жесткость. Такой кластер носит название «бесконечного» агрегата. В это время в реакционной среде еще много более мелких агрегатов и отдельных частиц золя, которые с течением времени присоединяются к «бесконечному» агрегату, что сопровождается увеличением его прочности. Точка $t_{2}$ " соответствует моменту времени, в который процессы образования и разрушения связей между частицами приходят к равновесию. Этот момент наступает тогда, когда все возможные малые агрегаты и частицы присоединились к «бесконечному» агрегату, и он имеет смысл времени полного перехода золя в гель. Далее, после точки $t_{2}{ }^{*}$ находится область старения геля.

Часть 2 статьи Кудрявцева П.Г., Фиговского О.Л. «Исследование золь-гель перехода реологическими методами» читайте в номере 5/2017 Интернет-журнала «Нанотехнологии в строительстве».

Список литературы к частям 1 и 2 статьи Кудрявцева П.Г., Фиговского О.Л. «Исследование золь-гель перехода реологическими методами» будет опубликован также в номере 5/2017 Интернет-журнала «Нанотехнологии в строительстве». 
УВАЖАЕМЫЕ КОЛЛЕГИ!

ПРИ ИСПОЛЬЗОВАНИИ МАТЕРИАЛА ДАННОЙ СТАТЬИ

ПРОСИМ ДЕЛАТЬ БИБЛИОГРАФИЧЕСКУЮ ССЫЛКУ НА НЕЁ:

Кудрявиев П.Г., Фиговский О.Л. Исследование золь-гель перехода реологическими методами. Часть I. Методы проведения экспериментов // Нанотехнологии в строительстве. - 2017. - Том 9, № 4. - C. 74-92. - DOI: dx.doi. org/10.15828/2075-8545-2017-9-4-74-92.

\section{Dear Colleagues!}

THE REFERENCE TO THIS PAPER HAS THE FOLLOWING CITATION FORMAT:

Kudryavtsev P.G., Figovsky O.L. Investigation of sol-gel transition by rheological methods. Part I. Experimental methods. Nanotehnologii v stroitel'stve = Nanotechnologies in Construction. 2017, Vol. 9, no. 4, pp. 74-92. DOI: dx.doi. org/10.15828/2075-8545-2017-9-4-74-92. (In Russian). 